\title{
Tendinopathy of the long head of the biceps tendon: histopathologic analysis of the extra-articular biceps tendon and tenosynovium
}

This article was published in the following Dove Press journal:

Open Access Journal of Sports Medicine

10 March 2015

Number of times this article has been viewed

\author{
Jonathan J Streit ${ }^{1}$ \\ Yousef Shishani' \\ Mark Rodgers ${ }^{2}$ \\ Reuben Gobezie' \\ 'The Cleveland Shoulder Institute, \\ ${ }^{2}$ Department of Pathology, University \\ Hospitals of Cleveland, Cleveland, \\ $\mathrm{OH}$, USA
}

Background: Bicipital tendinitis is a common cause of anterior shoulder pain, but there is no evidence that acute inflammation of the extra-articular long head of the biceps (LHB) tendon is the root cause of this condition. We evaluated the histologic findings of the extra-articular portion of the LHB tendon and synovial sheath in order to compare those findings to known histologic changes seen in other tendinopathies.

Methods: Twenty-six consecutive patients (mean age 45.4 \pm 13.7 years) underwent an open subpectoral biceps tenodesis for anterior shoulder pain localized to the bicipital groove. Excised tendons were sent for histologic analysis. Specimens were graded using a semiquantitative scoring system to evaluate tenocyte morphology, the presence of ground substance, collagen bundle characteristics, and vascular changes.

Results: Chronic inflammation was noted in only two of 26 specimens, and no specimen demonstrated acute inflammation. Tenocyte enlargement and proliferation, characterized by increased roundness and size of the cell and nucleus with proteoglycan matrix expansion and myxoid degenerative changes, was found in all 26 specimens. Abundant ground substance, collagen bundle changes, and increased vascularization were visualized in all samples.

Conclusion: Anterior shoulder pain attributed to the biceps tendon does not appear to be due to an inflammatory process in most cases. The histologic findings of the extra-articular portion of the LHB tendon and synovial sheath are similar to the pathologic findings in de Quervain tenosynovitis at the wrist, and may be due to a chronic degenerative process similar to this and other tendinopathies of the body.

Keywords: biceps tendinitis, biceps tendinopathy, tenosynovium, anterior shoulder pain, long head biceps tendon, histologic analysis

\section{Introduction}

The function of the long head of the biceps (LHB) tendon and its involvement in pain and disability of the anterior shoulder is widely debated and controversial. ${ }^{1-6}$ Recently, greater attention has been given to the intra-articular portion of the LHB tendon as a pain generator due to a better understanding of the changes that occur in association with inflammation, subluxation, dislocation, and rupture of the tendon., ${ }^{1,2,5,7-10}$ In addition, the extra-articular portion of the LHB tendon can also be a source of pain, and point tenderness in the bicipital groove on physical exam is often indicative of pain coming from this area. ${ }^{11,12}$ At the present time, it is unclear whether this pain is associated with acute inflammation or with chronic degenerative changes of the extraarticular LHB tendon. Several studies have evaluated the histopathologic changes of tendinopathies of the body, including the Achilles tendon, patellar tendon, supraspinatus
Correspondence: Reuben Gobezie The Cleveland Shoulder Institute, University Hospitals of Cleveland, 5885 Landerbrook Drive,

Monarch Center, Mayfield Heights, $\mathrm{OH} 44 \mathrm{I} 24$, USA

Email reuben.gobezie@uhhospitals.org
Open Access Journal of Sports Medicine 20I5:6 63-70 (c) (i) (-) 2015 Streit et al. This work is published by Dove Medical Press Limited, and licensed under Creative Commons Attribution - Non Commercial (unported, v3.0) BY LC License. The full terms of the License are available at http://creativecommons.org/licenses/by-nc/3.0/. Non-commercial uses of the work are permitted without any further permisssion from Dove Medical Press Limited, provided the work is properly attrib
request permission may be found at: http://www.dovepress.com/permissions.php 
tendon, lateral elbow extensor tendons, medial elbow flexor tendons, distal biceps tendon, intra-articular portion of the LHB tendon, and the abductor pollicis longus and extensor pollicis brevis within the first dorsal compartment of the wrist (de Quervain's disease). ${ }^{13-24}$ All of these tendons have been found to demonstrate similar histologic features of chronic degeneration, rather than acute inflammation. ${ }^{20,24-26}$ However, there have been no previous reports demonstrating the similarity of histologic findings between other tendinopathies of the body and those found in the extra-articular portion of the LHB tendon.

The purpose of this study was to perform a prospective histologic analysis of excised extra-articular biceps tendon taken from patients undergoing biceps tenodesis for relief of pain localized to the bicipital groove by physical exam. We hypothesized that the extra-articular LHB tendon and overlying tenosynovium sheath would exhibit histologic features of chronic degeneration, which would include changes in tenocyte morphology, mucoid degeneration, and extracellular matrix changes. We also hypothesized that these tendons would not exhibit acute inflammatory changes, making the commonly used term "tendinitis" a misnomer, as it is with many other tendinopathies of the body.

\section{Methods}

We prospectively identified 26 consecutive patients (mean age $45.4 \pm 13.7$ years; $63.3 \%$ male) undergoing biceps tenodesis and arthroscopic examination of the glenohumeral joint for pain that was localized to the bicipital groove by physical examination. Following the University Hospitals Case Medical Center institutional review board approval, each patient was offered inclusion in the study and provided informed consent to participate in the study prior to surgery. A diagnosis of biceps tendinitis/tendinosis was suspected preoperatively based on physical examination and radiographic findings. All physical examinations were performed by the senior author (RG), and this author's preoperative clinical examination included the Speed test, ${ }^{27} \mathrm{O}^{\prime}$ Brien's sign, ${ }^{28}$ and tenderness to palpation of the tendon within the bicipital groove at $10^{\circ}$ of internal rotation. ${ }^{11,12}$ The results of each of these maneuvers were recorded for later analysis. All patients also underwent a non-contrast MRI of the involved shoulder prior to surgery, which was read by the senior author as well as at least one musculoskeletal radiologist, prior to reaching the conclusion that the patient's anterior shoulder pain was likely to be caused, at least in part, by pathology of the LHB tendon.

All patients underwent a diagnostic shoulder arthroscopy as well as procedures performed for indications including subacromial impingement, rotator cuff tear, superior labral anterior-to-posterior tear, and osteoarthritis. All patients included in this study underwent arthroscopically assisted open subpectoral biceps tenodesis as part of their procedure. The senior author, who is a fellowship-trained shoulder surgeon, performed all surgeries, and all surgeries were performed in the beach chair position using a Biceps Button (Arthrex, Inc., Naples, FL, USA) for fixation within the distal aspect of the bicipital groove. A portion of the extra-articular LHB tendon located $2 \mathrm{~cm}$ proximal to the musculotendinous junction, which included both the sheath of tenosynovium as well as tendon substance, was excised and sent for histologic evaluation.

A single pathologist with experience evaluating musculoskeletal tissues performed the histologic analysis for each specimen. All tissue specimens were stained with hematoxylin and eosin (H\&E) and examined under light microscopy. Prior to examination, the pathologist defined the abnormal changes that would be noted: tendinopathy would be indicated by the presence of hypertrophic tenocytes with rounded nuclei, disorganized collagen bundles, and hypervascularity. The presence of inflammatory changes would be indicated by the presence of lymphocytes (small cells with minimal-tomoderate bluish cytoplasm and small, round-to-oval nuclei). Fibrosis would be identified by the presence of abnormally thick collagen within the tendon substance. Myxoid degeneration of the tendon would be defined as any area with loss of eosinophilic staining in the extracellular matrix and accumulation of amorphous, gray-blue material.

All specimens were evaluated for changes in tenocyte morphology, changes in the ground substance (noncollagenous component of the extracellular matrix), vascularity, and organization of the collagen bundles using both transmitted light microscopy and polarized light microscopy. The pathologist utilized a modified Bonar score ${ }^{15}$ to assess these characteristics of the tendon specimens (Table 1). A grade was given from 0 (normal appearance) to 3 (markedly abnormal appearance) for each of the four variables examined using light microscopy and H\&E staining: tenocyte morphology, ground substance, collagen, and vascularity. The grades from each variable were then summed, and a total score was given that ranged between 0 (normal tendon) and 12 (most severe abnormality detected). The Bonar score was originally used to classify the histopathologic findings of tendinopathy of the patellar tendon. Its reliability in classifying the histopathologic changes of tendinopathy, including the supraspinatus tendon, has been validated. ${ }^{29}$ Our modification of this semiquantitative scoring system involved the analysis of the 


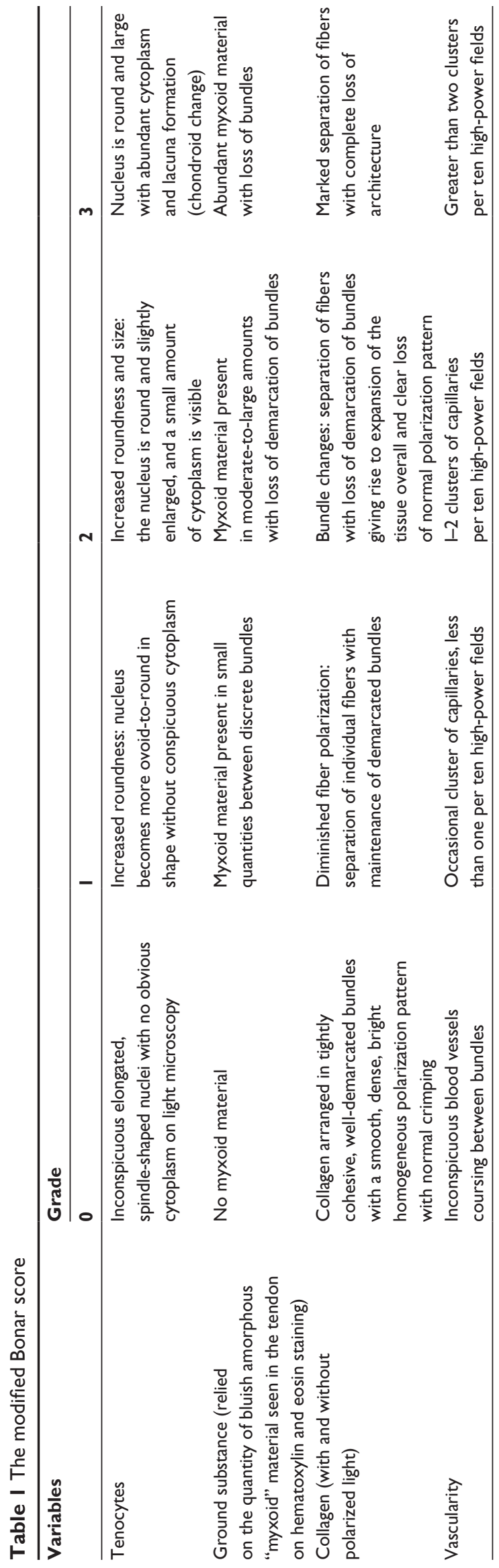

"ground substance" material. We did not use alcian blue and colloidal iron stains to quantify the amount of mucin between collagen bundles, as was originally described. ${ }^{15}$ Instead, we evaluated the amount of bluish, amorphous substance, or myxoid material, which is histologically similar to mucin when visualized on H\&E staining.

The sheath of tenosynovium was also examined. Hypercellularity, with prominence of synovial cells, redundancy and convolution of the synovial surface, and vascular proliferation, was also noted, and was determined to be indicative of synovial proliferation. The presence of abnormal vascularity was also noted within the tendon and synovial tissue. The severity of pathologic changes in the tenosynovium was then graded as mild, moderate, or severe, depending on the percent of involvement visualized during microscopy. Regarding the area of involvement, $0 \%-30 \%$ involvement was defined as mild, $30 \%-50 \%$ involvement was moderate, and greater than $50 \%$ involvement was severe.

\section{Results}

\section{Clinical data}

All patients underwent an open subpectoral biceps tenodesis for a complaint of pain, which could be localized to the anterior shoulder. Preoperative clinical examination showed that 93.3\% of patients demonstrated both a positive Speed test and positive O'Brien's sign. Tenderness over the tendon within the bicipital groove at the anterior aspect of the proximal humerus was present in $100 \%$ of patients.

By arthroscopy, there was evidence of a superior labral anterior-to-posterior tear in $86.7 \%$ of patients, with $42.3 \%$ of these having a type I tear and $57.7 \%$ having a type II tear. A tear of the biceps tendon distal to its root was identified in $33.3 \%$ of patients. In addition, $66 \%$ underwent subacromial decompression, $33.3 \%$ underwent distal clavicle resection, and $26.7 \%$ underwent rotator cuff repair.

\section{Histologic data}

All specimens expressed morphologic changes of tenocytes, noted by increase in size and roundness of the cell and nucleus (Figure 1). Ground substance, collagen bundle changes, and vascular changes were noted in all samples and were graded according to the modified Bonar scale (Table 2). Grade 2 or 3 morphologic changes of the tenocytes were identified in 24 of 26 specimens (93\%). Ground substance was visualized in all specimens, and 25 of 26 specimens (96\%) were determined to have grade 2 changes (Figure 2). Twenty specimens demonstrated grade 2 collagen bundle characteristics (Figures 3 and 4). Abnormal clusters of capillaries were 


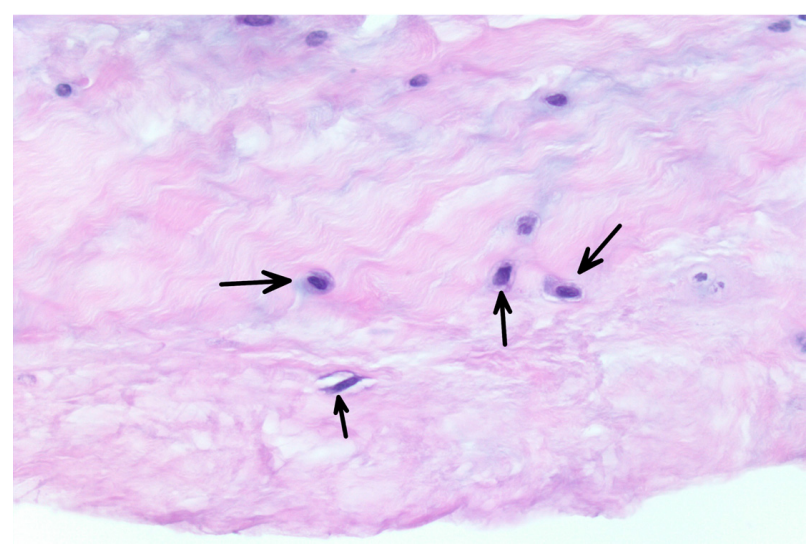

Figure I The tenocyte nuclei are enlarged and rounded (arrows), and the cells show a small amount of visible cytoplasm.

identified in all samples, with $58.6 \%$ scored as grade 2 vascularity and $41.4 \%$ scored as grade 1 vascularity (Figure 5).

The specimens recovered from our patient group exhibited an overall trend toward degeneration in the tendon substance, without associated acute inflammation. Histologic analysis of the biceps tendon substance revealed evidence of mild chronic inflammation in two of $26(7.7 \%)$ specimens. There was no moderate or severe inflammation identified in any specimen.

Table 2 Results of tendon pathologic scores using the modified Bonar score $(\mathrm{N}=26)$

\begin{tabular}{llllll}
\hline Specimen & Tenocytes & $\begin{array}{l}\text { Ground } \\
\text { substance }\end{array}$ & Collagen & Vascularity & $\begin{array}{l}\text { Total } \\
\text { score }\end{array}$ \\
\hline 1 & 2 & 2 & 2 & 2 & 8 \\
2 & 2 & 2 & 2 & 2 & 8 \\
3 & 3 & 2 & 2 & 1 & 8 \\
4 & 3 & 2 & 2 & 1 & 8 \\
5 & 2 & 2 & 2 & 2 & 8 \\
6 & 2 & 2 & 3 & 1 & 8 \\
7 & 2 & 2 & 2 & 2 & 8 \\
8 & 2 & 2 & 2 & 2 & 8 \\
9 & 3 & 2 & 2 & 1 & 8 \\
10 & 2 & 2 & 2 & 2 & 8 \\
11 & 2 & 2 & 2 & 1 & 7 \\
12 & 2 & 2 & 2 & 1 & 7 \\
13 & 2 & 2 & 2 & 2 & 8 \\
14 & 2 & 2 & 2 & 1 & 7 \\
15 & 2 & 2 & 1 & 1 & 6 \\
16 & 2 & 2 & 1 & 1 & 6 \\
17 & 2 & 2 & 2 & 2 & 8 \\
18 & 2 & 2 & 3 & 2 & 9 \\
19 & 2 & 2 & 2 & 1 & 7 \\
20 & 3 & 2 & 1 & 1 & 7 \\
21 & 3 & 2 & 2 & 2 & 9 \\
22 & 3 & 2 & 1 & 3 & 9 \\
23 & 1 & 1 & 1 & 2 & 5 \\
24 & 1 & 2 & 2 & 2 & 7 \\
25 & 2 & 2 & 2 & 2 & 8 \\
26 & 2 & 2 & 1 & 1 & 6 \\
\hline
\end{tabular}

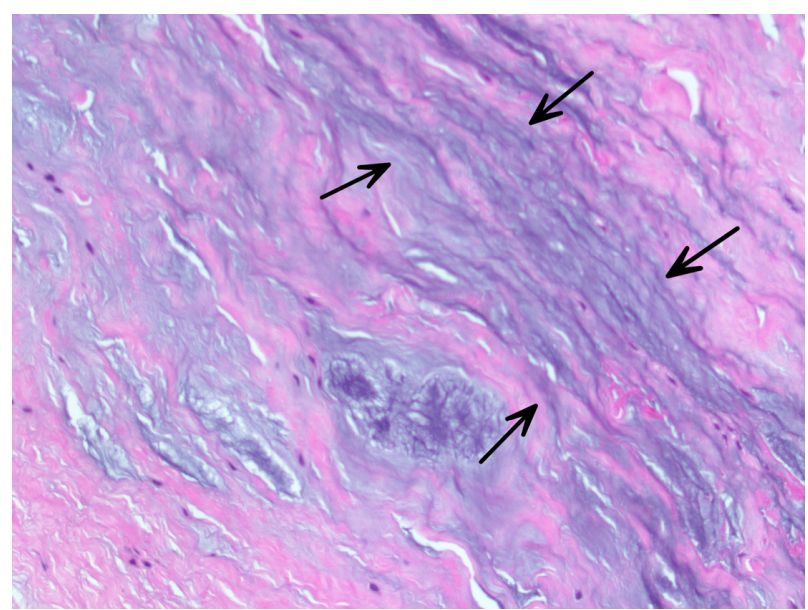

Figure 2 Myxoid ground substance, visible as blue-gray amorphous material (arrows), separating collagen bundles within a hematoxylin and eosin-stained section.

Myxoid degeneration was identified in all specimens, with three showing mild changes, 20 showing moderate changes, and three demonstrating severe changes. Fibrosis of the tendon was identified in all 26 specimens; 16 specimens demonstrated mild fibrosis, ten demonstrated moderate fibrosis, and none showed evidence of severe fibrosis.

Histologic analysis of the sheath tenosynovium revealed synovial proliferation without any evidence of acute or chronic inflammation in any subject (Figure 6). There was synovial proliferation evident in 14/30 specimens, of which 12 demonstrated mild proliferation and two demonstrated moderate proliferation.

\section{Discussion}

The anatomy of the LHB tendon is unique in that it has both intra- and extra-articular portions, each exposed to

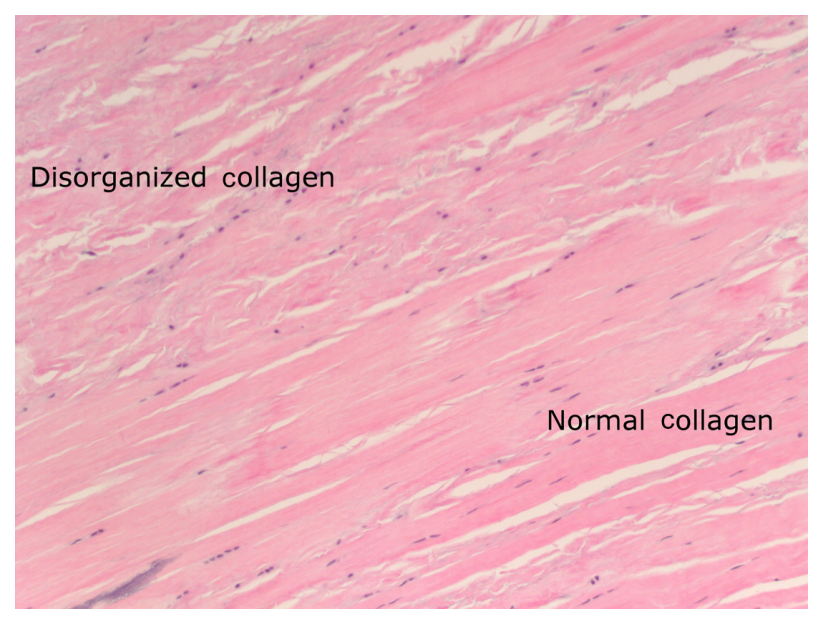

Figure 3 Normally organized collagen (lower right) and disorganized collagen showing separation, fragmentation, and disorientation of fibers (upper left of image). 


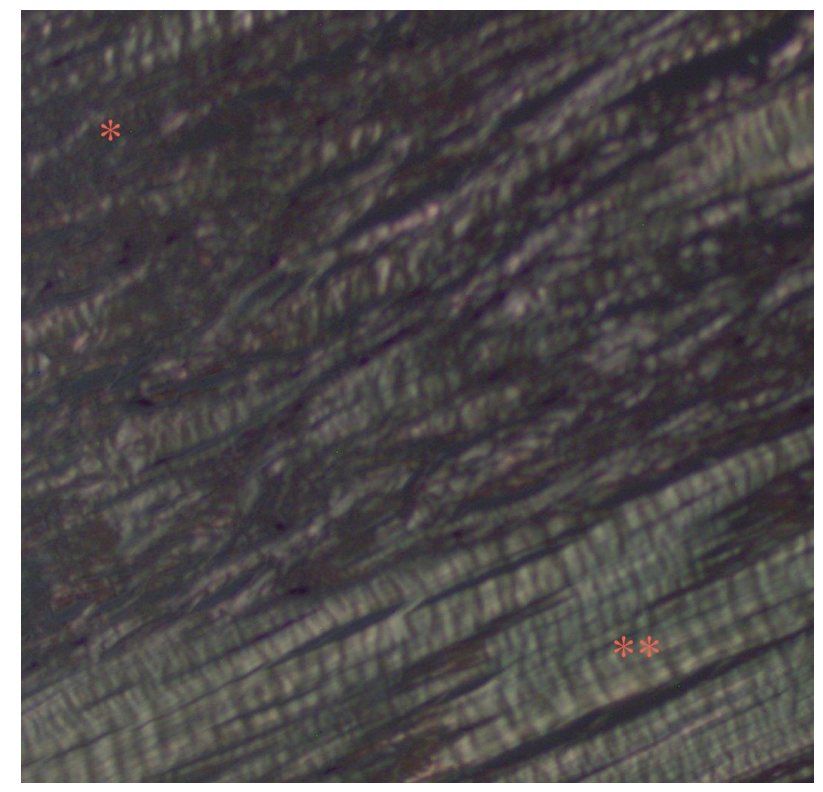

Figure 4 Polarized light microscopy shows areas of fragmentation of the collagen and loss of normal polarization pattern $\left(^{*}\right)$.

Notes: Areas with an appearance more typical of intact collagen arranged in tightly cohesive and well-demarcated bundles with a homogeneous polarization pattern are also visualized (**).

different loading patterns. ${ }^{1,3,4,30}$ While tenotomy and tenodesis procedures are often successful treatments for relieving anterior shoulder pain associated with intra-articular pathology, ${ }^{1,2,4,6,30-33}$ the true pathophysiology of extra-articular biceps-related pain remains poorly understood. ${ }^{2,5}$ Anatomically, the tendon is contained within a synovial sheath as it passes through the shoulder joint, and this sheath continues to cover the tendon through the bicipital groove. ${ }^{2,4,5,30}$ While most studies of the LHB tendon have focused on the intraarticular portion, especially in conjunction with rotator cuff tears, there are limited data concerning the pathologic changes of the extra-articular biceps tendon. ${ }^{4,5,6,10,30,33,34}$

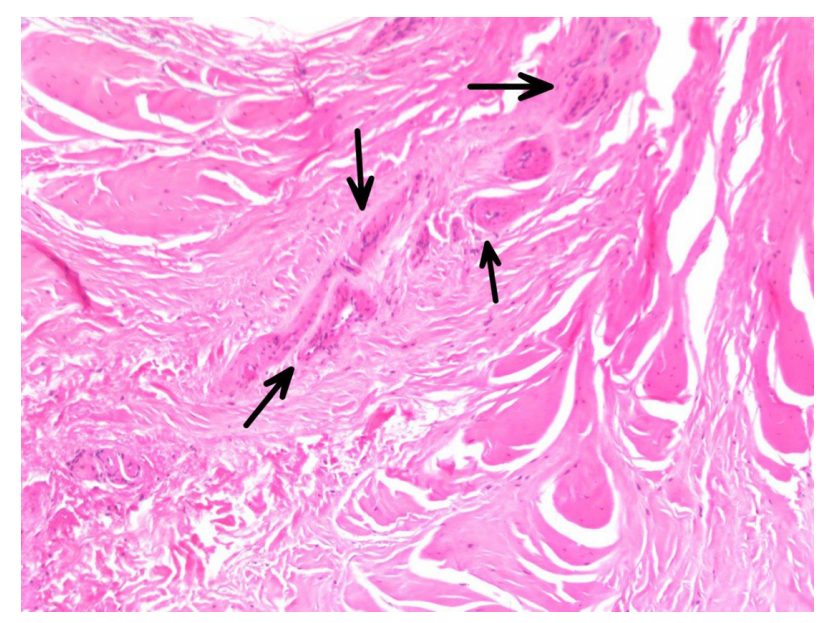

Figure $\mathbf{5}$ Vascular proliferation with clusters of capillaries visualized (arrows).

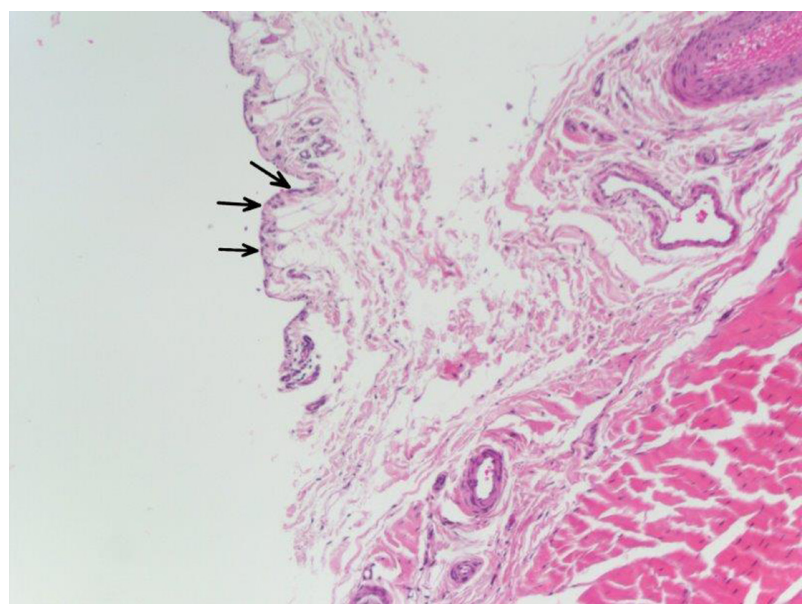

Figure 6 This histologic image of the long head of the biceps tenosynovium demonstrates reactive features, including synovial proliferation, enlargement of surface synovial cells (arrows), and vascular proliferation.

Note: There is an absence of neutrophils, indicating a physiologic response in the absence of acute inflammation.

Our hypothesis that the extra-articular LHB tendon and sheath tenosynovium would closely resemble those histopathologic changes consistent with other tendinopathies of the body was supported by our findings. Microscopic analysis revealed disorganized collagen bundles, increased ground substance consisting of proteoglycans and glycosaminoglycans, rounded tenocytes and nuclei, and increased vascularity. These are well-established histopathologic changes seen in chronic tendinopathies affecting other parts of the body. ${ }^{26}$

The histologic features of tendinosis/tendinopathy or tendinitis have been examined at multiple anatomic locations, and all exhibit similar pathologic findings, involving changes in tenocyte morphology and number with changes to the extracellular matrix..$^{11,13,15,20,24,26,35}$ Riley $^{20}$ and $\mathrm{Xu}$ and Murrell ${ }^{26}$ emphasize the importance of tenocyte enlargement and proliferation with matrix expansion due to increased proteoglycan production and subsequent expression of cytokines, growth hormones, and signaling pathways in the development of tendinitis. Changes to the tendon progenitor stem cells, which occur when a tendon is exposed to altered loads, have also been implicated in the histopathologic changes seen in tendinopathy. ${ }^{24,35}$ Mechanical loading of tendons has been shown to be detrimental by directing differentiation of tenocyte stem cells into adipogenic, osteogenic, and chondrogenic lineages within the tendon, resulting in lipid accumulation, mucoid formation, and tissue calcification. ${ }^{24}$ In an animal model, Zhang and Wang ${ }^{23}$ showed that high levels of prostaglandin E2, which is present in tendons subjected to high stress, would result in degenerative changes to the tendon by decreasing the amount of tenocyte stem cells available for repair of the tendon and inducing differentiation into 
adipocytes and osteocytes. They concluded that this might lead to the production of fatty and calcified tissues within the tendon, which is often seen at later stages of tendinopathy. ${ }^{24}$ Such changes are deleterious to the tendon and explain the increased proteoglycan production and collagen degeneration that has been documented in our study. As such, it is possible that the changes seen in biceps tendinopathy are due to an adaptive phenomenon and an alteration of cellular activity.

Tendon myxoid degeneration and fibrosis was a common finding in our study. Previous studies evaluating the intra-articular portion of the biceps tendon in patients with anterior shoulder pain have pointed to the presence of myxoid degeneration in the biceps tendon. ${ }^{10,25,30,36,37}$ Singaraju et $\mathrm{al}^{6}$ showed low levels of inflammatory cells in the intra-articular biceps tendon of patients with anterior shoulder pain and presumed biceps tendinitis. They also found no correlation between inflammation of the biceps tendon and anterior shoulder pain in their patient population. Longo et $\mathrm{a}^{25}$ found marked degenerative changes of the intra-articular portion of the LHB tendon in patients undergoing arthroscopic tenotomy for tears of $>50 \%$ of the tendon found at the time of arthroscopy. These changes included an increase in the number of tenocytes with rounded nuclei and a disordered arrangement of collagen fibers. There was an absence of inflammatory changes seen histopathologically in their study as well, indicating that there would be a poor healing response of the tendon. ${ }^{25}$

The terms "tendinitis", "tendinosis", and "tendinopathy" are often used interchangeably to describe pain emanating from a tendon that often results from overuse. However, "tendinopathy" is better suited to describing chronic pain affecting a tendon and is used in preference to terms such as "tendinosis" or "tendinitis", as this terminology makes no assumption of the underlying pathology. ${ }^{20}$ Tendinopathies are primarily degenerative conditions with an absence of inflammatory cells, although the role of inflammation is still debated..$^{20}$ The term "tendinitis" of the LHB tendon is commonly used to describe anterior shoulder pain resulting from repetitive motion that occurs as the tendon courses along a constrained path within the bicipital groove. ${ }^{4}$ The results of our study indicate that no inflammatory process actually causes chronic extra-articular biceps tendon pain. As such, we propose that the term "biceps tendinopathy" is better suited to describe this condition, as opposed to "biceps tendinitis".

Because of the LHB tendon's course in the bicipital groove surrounded by tenosynovium and the overlying transverse humeral ligament, we feel that the pathologic changes affecting this tendon are best conceptualized as being similar to those occurring within the first dorsal compartment of the wrist in de Quervain tenosynovitis. The histopathologic changes of de Quervain's disease were noted in 1998 by Clarke et $\mathrm{al}^{38}$ to be attributed to thickening of the tendon sheath with dense fibrous tissue and accumulation of mucopolysaccharide, implicating intrinsic myxoid degeneration, rather than extrinsic inflammation, in the pathophysiology of the disease. ${ }^{38}$ The authors excised only the tendon sheath, as this is the standard treatment for de Quervain's disease, and did not evaluate the tendon substance. They found myxoid degenerative changes within the fibrous layer of the tendon sheath, but not in the synovial lining, with increased vascularity throughout. ${ }^{38}$ Because the fibrous layer of the tendon sheath is in intimate contact with the tendon, it might be assumed that similar changes of myxoid degeneration would be present within the tendon itself. The results of our study show that myxoid degeneration was universally present in our excised tendons, and fibrosis was almost always present. Additionally, our study also shows that synovial proliferation without inflammatory changes within the tenosynovium mimics the tendon sheath changes found in de Quervain's disease. ${ }^{38}$

The findings of our study make sense in light of recent investigations of biceps-related shoulder pain. Previous studies have shown that chronic inflammation of the biceps tendon is a common occurrence in the setting of rotator cuff tears. ${ }^{2,5,34}$ Murthi et $\mathrm{al}^{34}$ have previously reported on both chronic inflammation and degeneration of the extraarticular biceps tendon in patients with rotator cuff tears, the extent of which was associated with the severity of rotator cuff tearing. The authors showed chronic inflammatory changes in $63 \%$ of shoulders with partial-thickness tears and in $75 \%$ of shoulders with full-thickness tears. Chronic inflammation of the tendon sheath was also noted in $53 \%$ of shoulders without rotator cuff tears, ${ }^{34}$ although this finding of chronic inflammatory changes of the bicipital tendon sheath was not reproduced in our study. A recent study by Sanders et $\mathrm{al}^{39}$ on the results of biceps tenodesis, based on location of the tenodesis, demonstrated a strong association between relief of pain and release of the transverse humeral ligament. They showed a significant decrease in reoperation rates among patients treated with release of the transverse humeral ligament or a distal tenodesis, compared to proximal tenodesis not decompressing the extra-articular biceps tendon. Release of the transverse humeral ligament directly mimics the treatment for de Quervain's disease at the wrist. ${ }^{38}$ We believe that the findings of our study lend support to the idea that the extra-articular biceps tendon may be more of 
a pain generator than previously thought, due to a chronic stenosing degenerative pathologic process surrounding the LHB within the biceps groove. It is possible that pain localized to the bicipital groove with shoulder flexion represents tendon motion similar to that which occurs at the first dorsal compartment of the wrist with the provocative Finkelstein test in patients with de Quervain's disease. ${ }^{38}$

Although we believe our findings are valuable, there are limitations to our study. We did not include a control group for histologic comparison, and it is possible that some biceps tendons that were not painful would have also demonstrated degenerative changes. Also, a single pathologist performed histopathologic analysis for this study. Interobserver analysis may have allowed us to differentiate the severity of pathologic changes related to the scoring system and provided information regarding the reliability of the modified Bonar score. However, the reliability of this scoring system has been validated for both tendinopathies of the upper and lower extremities. ${ }^{15,29}$ Another limitation to our study was the inability to isolate patients with only bicipital groove pain without other shoulder pathology. However, anterior shoulder pain due to isolated extra-articular biceps tendinopathy is uncommon, and is often found in the presence of associated subacromial impingement and intra-articular shoulder pathology. We feel that our study population comprises a representative sample of patients who would normally present to an orthopedic surgeon's office for treatment of anterior shoulder pain. Future studies involving the histologic analysis of both normal cadaveric tendons and tendons from individuals with shoulder pain may further refine our understanding of the pathophysiology of extra-articular biceps pain. Future basic science studies may focus on the pathways leading to the formation of ground substance, myxoid degeneration, and vascular proliferation. It is possible that a single underlying process, such as increased stress on the tendon, may be the cause, as our pathologist has noted similar histologic changes in heart valves and other fibroconnective tissues elsewhere in the body.

\section{Conclusion}

The results of our histologic analysis of painful extra-articular biceps tendons and tendon sheaths suggest that biceps tendinopathy is similar histologically to other tendinopathies of the human body. We propose that extra-articular biceps pain may not be due to an inflammatory condition, but rather to a degenerative process resulting from repetitive motion within the bicipital groove. As such, treatment of extra-articular biceps pathology should attempt to resolve the chronic compression and instability of the tendon, and it is possible that release of the transverse humeral ligament and a distal tenodesis provides a more complete treatment for this condition. A better understanding of the pathologic changes outlined in this study may also lead to novel methods in the treatment of biceps-related anterior shoulder pain.

\section{Disclosure}

Dr R Gobezie is a consultant for Arthrex Inc. and receives royalties. The authors report no other conflicts of interest in this work.

\section{References}

1. Barber A, Field LD, Ryu R. Biceps tendon and superior labrum injuries: decision-marking. J Bone Joint Surg Am. 2007;89(8):1844-1855.

2. Khazzam M, George MS, Churchill RS, Kuhn JE. Disorders of the long head of biceps tendon. J Shoulder Elbow Surg. 2012;21(1):136-145.

3. McGough RL, Debski RE, Taskiran E, Fu FH, Woo SL. Mechanical properties of the long head of the biceps tendon. Knee Surg Sports Traumatol Arthrosc. 1996;3(4):226-229.

4. Nho SJ, Strauss EJ, Lenart BA, et al. Long head of the biceps tendinopathy: diagnosis and management. J Am Acad Orthop Surg. 2010;18(11):645-656.

5. Sethi N, Wright R, Yamaguchi K. Disorders of the long head of the biceps tendon. J Shoulder Elbow Surg. 1999;8(6):644-654.

6. Singaraju VM, Kang RW, Yanke AB, et al. Biceps tendinitis in chronic rotator cuff tears: a histologic perspective. J Shoulder Elbow Surg. 2008;17(6):898-904.

7. Boileau P, Ahrens PM, Hatzidakis AM. Entrapment of the long head of the biceps tendon: the hourglass biceps - a cause of pain and locking of the shoulder. J Shoulder Elbow Surg. 2004;13(3):249-257.

8. Kelly AM, Drakos MC, Fealy S, Taylor SA, O'Brien SJ. Arthroscopic release of the long head of the biceps tendon: functional outcome and clinical results. Am J Sports Med. 2005;33(2):208-213.

9. Post M, Benca P. Primary tendinitis of the long head of the biceps. Clin Orthop Relat Res. 1989;(246):117-125.

10. Refior HJ, Sowa D. Long tendon of the biceps brachii: sites of predilection for degenerative lesions. J Shoulder Elbow Surg. 1995;4(6): 436-440.

11. Longo UG, Loppini M, Marineo G, Khan WS, Maffulli N, Denaro V. Tendinopathy of the tendon of the long head of the biceps. Sports Med Arthrosc. 2011;19(4):321-332.

12. Patton WC, McCluskey GM 3rd. Biceps tendinitis and subluxation. Clin Sports Med. 2001;20(3):505-529.

13. Aström M, Rausing A. Chronic Achilles tendinopathy. A survey of surgical and histopathologic findings. Clin Orthop Relat Res. 1995;(316) 151-164.

14. Aström M, Westlin N. Blood flow in chronic Achilles tendinopathy. Clin Orthop Relat Res. 1994;(308):166-172.

15. Cook JL, Feller JA, Bonar SF, Khan KM. Abnormal tenocyte morphology is more prevalent than collagen disruption in asymptomatic athletes' patellar tendons. J Orthop Res. 2004;22(2):334-338.

16. Cook JL, Purdam C. Is compressive load a factor in the development of tendinopathy? Br J Sports Med. 2012;46(3):163-168.

17. Henton J, Jain A, Medhurst C, Hettiaratchy S. Adult trigger finger. $B M J$. 2012;345:e5743.

18. Neal BS, Longbottom J. Is there a role for acupuncture in the treatment of tendinopathy? Acupunct Med. 2012;30(4):346-349.

19. Ribbans WJ, Collins M. Pathology of the tendo Achillis: do our genes contribute? Bone Joint J. 2013;95-B(3):305-313. 
20. Riley G. Tendinopathy - from basic science to treatment. Nat Clin Pract Rheumatol. 2008;4(2):82-89.

21. Scott A, Cook JL, Hart DA, Walker DC, Duronio V, Khan KM. Tenocyte responses to mechanical loading in vivo: a role for local insulin-like growth factor 1 signaling in early tendinosis in rats. Arthritis Rheum. 2007;56(3):871-881.

22. Stevens K, Kwak A, Poplawski S. The biceps muscle from shoulder to elbow. Semin Musculoskelet Radiol. 2012;16(4):296-315.

23. Zhang J, Wang JH. Production of PGE(2) increases in tendons subjected to repetitive mechanical loading and induces differentiation of tendon stem cells into non-tenocytes. J Orthop Res. 2010;28(2):198-203.

24. Zhang J, Wang JH. Mechanobiological response of tendon stem cells: implications of tendon homeostasis and pathogenesis of tendinopathy. J Orthop Res. 2010;28(5):639-643.

25. Longo UG, Franceschi F, Ruzzini L, et al. Characteristics at haematoxylin and eosin staining of ruptures of the long head of the biceps tendon. Br J Sports Med. 2009;43(8):603-607.

26. Xu Y, Murrell GA. The basic science of tendinopathy. Clin Orthop Relat Res. 2008;466(7):1528-1538.

27. Neviaser RJ. Lesions of the biceps and tendinitis of the shoulder. Orthop Clin North Am. 1980;11(2):343-348.

28. O’Brien SJ, Pagnani MJ, Fealy S, McGlynn SR, Wilson JB. The active compression test: a new and effective test for diagnosing labral tears and acromioclavicular joint abnormality. Am J Sports Med. 1998;26(5): 610-613.

29. Maffulli N, Longo UG, Franceschi F, Rabitti C, Denaro V. Movin and Bonar scores assess the same characteristics of tendon histology. Clin Orthop Relat Res. 2008;466(7):1605-1611.

30. Joseph M, Maresh CM, McCarthy MB, et al. Histological and molecular analysis of the biceps tendon long head post-tenotomy. J Orthop Res. 2009;27(10):1379-1385.
31. Boileau P, Baqué F, Valerio L, Ahrens P, Chuinard C, Trojani C. Isolated arthroscopic biceps tenotomy or tenodesis improves symptoms in patients with massive irreparable rotator cuff tears. $J$ Bone Joint Surg Am. 2007;89(4):747-757.

32. Frost A, Zafar MS, Maffulli N. Tenotomy versus tenodesis in the management of pathologic lesions of the tendon of the long head of the biceps brachii. Am J Sports Med. 2009;37(4):828-833.

33. Walch G, Edwards TB, Boulahia A, Nové-Josserand L, Neyton L, Szabo I. Arthroscopic tenotomy of the long head of the biceps in the treatment of rotator cuff tears: clinical and radiographic results of 307 cases. J Shoulder Elbow Surg. 2005;14(3):238-246.

34. Murthi AM, Vosburgh CL, Neviaser TJ. The incidence of pathologic changes of the long head of the biceps tendon. J Shoulder Elbow Surg. 2000;9(5):382-385.

35. Bi Y, Ehirchiou D, Kilts TM, et al. Identification of tendon stem/ progenitor cells and the role of the extracellular matrix in their niche. Nat Med. 2007;13(10):1219-1227.

36. Berenson MC, Blevins FT, Plaas AH, Vogel KG. Proteoglycans of human rotator cuff tendons. J Orthop Res. 1996;14(4):518-525.

37. Kannus P, Józsa L. Histopathological changes preceding spontaneous rupture of a tendon. A controlled study of 891 patients. J Bone Joint Surg Am. 1991;73(10):1507-1525.

38. Clarke MT, Lyall HA, Grant JW, Matthewson MH. The histopathology of de Quervain's disease. J Hand Surg Br. 1998;23(6):732-734.

39. Sanders B, Lavery KP, Pennington S, Warner JJ. Clinical success of biceps tenodesis with and without release of the transverse humeral ligament. J Shoulder Elbow Surg. 2012;21(1):66-71.
Open Access Journal of Sports Medicine

\section{Publish your work in this journal}

Open Access Journal of Sports Medicine is an international, peer-reviewed, open access journal publishing original research, reports, reviews and commentaries on all areas of sports medicine. The manuscript management system is completely online and includes a very quick and fair peer-review system.

\section{Dovepress}

Visit http://www.dovepress.com/testimonials.php to read real quotes from published authors. 